\title{
Epileptic Seizure Prediction using Statistical Behavior of Local Extrema and Fuzzy Logic System
}

\author{
Hamid Niknazar \\ Department of Biomedical \\ Engineering, Science and \\ Research Branch, Islamic \\ Azad University, Tehran, Iran
}

\author{
Keivan Maghooli \\ Department of Biomedical \\ Engineering, Science and \\ Research Branch, Islamic \\ Azad University, Tehran, Iran
}

\author{
Ali Motie Nasrabadi \\ Department of Biomedical \\ Engineering Shahed \\ University, Tehran, Iran
}

\begin{abstract}
Epileptic seizures are generated by abnormal activity of neurons. EEG-based epileptic seizure prediction could be a key to improve life style of patients that suffer from drugresistance epilepsy. In this study, we propose a fuzzy logic system to predict epileptic seizures by using statistical behavior of local extrema (SBLE) features and a rule-based fuzzy system. Two approaches are considered to evaluate the proposed method. First approach is patient-dependent, which requires EEG data in preictal and interictal state. Second approach is leave one out (LOO) technique to evaluate generalizability of the method. Applied to the Freiburg EEG dataset, it was found that the method has good performance for most of the patients of this database. In the patientdependent approach, sensitivity of $84 \%$ with no false alarm and sensitivity of $94.15 \%$ with a false alarm rate of 0.1 were achieved. LOO evaluation approach obtained a sensitivity of $79.38 \%$ with a false alarm rate of 0.049 . It is remarkable that for many of patients, the proposed method achieved sensitivity of $100 \%$ with no false alarm in both of evaluation approaches. This study showed that application of SBLE features as inputs of fuzzy logic system is a suitable way to track EEG changes leading to epileptic seizures.
\end{abstract}

\section{Keywords}

Epilepsy, Fuzzy Logic, SBLE, Prediction, Genetic Algorithm

\section{INTRODUCTION}

Epilepsy is a neurological disease that is characterized by recurrent and abrupt seizures affecting millions of individuals worldwide [1]. Patients with uncontrolled epilepsy may suffer from unwanted side effects such as memory loss, depression and other psychological disorders. They can also be hurt by accidents caused by unforeseen seizures as well as sudden unexpected death [2]. Despite advances in anti-epileptic medications, drug resistant epilepsy still lacks an ultimate solution [3]. The outcome of a resective surgery, where the part of the brain that causes the seizures is removed [4] is highly unpredictable. Moreover, this solution can be only applied to a small population of drug-resistant patients. Additionally, the cause of drug-resistance epilepsy is still unknown.

Epilepsy can be studied by analyzing electroencephalogram (EEG) signals, since it is a condition related to the electrical activity of brain. As a result, several mathematical and statistical techniques based on EEG signal processing, have been proposed to improve the performance of epileptic seizure prediction and detection [5].

In 1975 the first work on prediction and detection of epileptic seizures was done by Viglione and Walsh. They used a linear approach to find seizure precursors [6]. Rogowski et al. [7] and then Salant et al. [8] used an autoregressive model to find changes prior seizure onsets. Iaesemidis et al. [9] employed the Lyapunov exponent and an open window analysis and revealed a decrease in chaotic behavior of EEG signal before seizures. In recent studies, Costa et al. [10] used various neural networks for classifying EEG recordings into preictal (moments before seizure), ictal (seizure period), post-ictal (moments after seizure), and interictal (period between postictal and preictal) classes. They reported a sensitivity of $98.5 \%$, specificity of $99.5 \%$, and accuracy of $98.5 \%$ on EEG recordings of two patients from the Freiburg EEG database [11]. Moghim et al. [12] used some features based on energy, discrete wavelet transforms and nonlinear dynamics and multi-class support vector machine (SVM) classifier and reported an accuracy of $97.68 \%$, specificity of $99.55 \%$, and sensitivity of $91.14 \%$ on all the patients of Freiburg database. Ghaderyan et al. [13] utilized 6 features that are extracted from time and frequency domains and principle component analysis (PCA) for feature selection. By using SVM classifier for classification, they reported a sensitivity of $76.94 \%$ and specificity of $87.76 \%$ on all the 21 patients of Freiburg EEG database.

The large number of algorithms found in seizure prediction literature can be classified into several broad categories. First of all, most of the methods developed are based on a threshold technique on the output value of a seizure prediction method, such as phase synchronization [14]. Another group of study applied clustering based techniques to classify preictal and interictal classes [15]. This group used a machine learning based approaches such as artificial neural network (ANN), support vector machine (SVM) or neuro-fuzzy system (ANFIS) classifiers with multiple features that were extracted from EEG signal [16], [17]. This approach is supervised and needs training from preictal and interictal datasets. Recently, a patient specific rule-based approach on combination of spatial and temporal domain features was proposed [18]. A fuzzy rule-based system was also proposed for epileptic seizure detection from intracranial EEG for taking advantage of the combination in the feature domain as well as in the spatial domain [19]. And recently, adaptive neuro-fuzzy inference system (ANFIS) was used to combine multiple epileptic seizure predictive features: nonlinear univariate and bivariate [20]. This study reported a sensitivity of $80 \%$ with 0.46 false positive rate (FPR).

This paper presents the application of fuzzy logic system in epileptic seizure prediction. We applied a fuzzy logic system to combine the statistical behavior of local extrema (SBLE) features for identifying the preictal state. Fuzzy logic system efficiently performs a nonlinear input output mapping by considering the complex relationships of the feature space. The rest of this paper is organized as follows. Section II is devoted to describe the dataset in detail. Materials and methods are presented in Section III. The result of the 
proposed method applied on the dataset and discussion about the results are provided in Section IV. Finally, our conclusions are stated in Section V.

\section{DATABASE}

The Freiburg EEG database 2007 [11] is used in this study to evaluate the performance of the proposed method. This dataset contains invasive EEG recordings of 21 patients suffering from medically intractable focal epilepsy. The data were recorded at the Epilepsy Center of the University Hospital of Freiburg. The EEG data are available on 6 channels at $256 \mathrm{~Hz}$ sampling rate. Figure 1 shows an example of placing electrods.

For each of the patients, there are datasets named "ictal" and "interictal", the former containing files with epileptic seizures and at least $54 \mathrm{~min}$ preictal data and the latter containing approximately 24 hours of EEG-recordings without seizure activity.

\section{MATERIALS AND METHOD}

In the proposed method some features that extracted by SBLE method are used to construct a fuzzy logic decision-making machine. 15 features are extracted by SBLE method then membership functions are estimated from training dataset, then by using a genetic algorithm efficient fuzzy rules are constructed. Proposed method consists of five steps:

1) Preprocessing

2) Features extraction

3) Designing fuzzy logic system that consists of two steps:

a) Estimating membership functions

b) Extracting efficient fuzzy rules

4) Post-processing

5) Decision making

\subsection{Preprocessing}

Database is divided to two datasets, training and testing. Training dataset consists of $20 \%$ of interictal signal and preictal signal of one seizure for each patient. All patients have at least 24 hours interictal and 2 to 5 seizures, so for each patient training dataset consists of about 5 hours interictal and about one hour preictal EEG data. Therefore, about 19 hours interictal and 1 to 4 hours preictal EEG data for each patient builds the testing dataset.

Two filters are used to reduce noises and artifacts. First, a 50 $\mathrm{Hz}$ notch filter is used to remove power line noise. Then, a 4$30 \mathrm{~Hz}$ band-pass filter is used to remove baseline and high frequency artifacts.

\subsection{Features Extraction}

SBLE method proposed 15 features that characterize behavior of sequential local extrema. These features are used as the input of fuzzy logic system. A 5 second (1280 samples) windowing technique with no overlap is used. The features are extracted for each windowed signal $T_{n}$ ( $n$ is window index). First, three ranges are defined to extract features as:

$$
\begin{aligned}
& \left.\mathrm{R}_{1}\right) \mathrm{D}_{\mathrm{T}_{\mathrm{n}}}>\mu+\sigma \\
& \left.\mathrm{R}_{2}\right) \mu-\sigma<\mathrm{D}_{\mathrm{T}_{\mathrm{n}}}<\mu+\sigma \\
& \left.\mathrm{R}_{3}\right) \mathrm{D}_{\mathrm{T}_{\mathrm{n}}}<\mu-\sigma
\end{aligned}
$$

where $D_{T_{n}}$ is amplitude of $\mathrm{T}_{\mathrm{n}}, \mu$ and $\sigma$ are mean and standard deviation of the $T_{n}$ windowed signal.

Then, local extrema of $T_{n}$ are extracted. Before introducing features, it is necessary to define some patterns that are shown in Figure 1:

- P1) Increase pattern: existence of $\mathrm{s}_{\mathrm{n}-1} \mathrm{~s}_{\mathrm{n}}$ sequence, where:

$\circ s_{n-1} \in R_{i}, s_{n} \in R_{j}$ and $i>j$ (Figure 1(a)).

- P2) Decrease pattern: existence of $s_{n-1} s_{n}$ sequence, where:

$\circ s_{n-1} \in R_{i}, s_{n} \in R_{j}$ and $i<j$ (Figure 1(b)).

- P3) U-turn1 pattern: existence of $\mathrm{s}_{\mathrm{n}-2} \mathrm{~s}_{\mathrm{n}-1} \mathrm{~s}_{\mathrm{n}}$ sequence, where:

$\circ \quad s_{n-2} \in R_{i}, s_{n-1} \in R_{j}, s_{n} \in R_{k}$ and $i>j, j<k$ (Figure 1(c)).

- P4) U-turn2 pattern: existence of $s_{n-2} s_{n-1} s_{n}$ sequence, where:

$\circ \quad s_{n-2} \in R_{i}, s_{n-1} \in R_{j}, s_{n} \in R_{k}$ and $i<j, j>k$ (Figure 1(d)).

- P5) Increase-Constant pattern: existence of $s_{n-2} s_{n-1} s_{n}$ sequence, where:

○ $s_{n-2} \in R_{i}, s_{n-1} \in R_{j}, s_{n} \in R_{k}$ and $i>j, j=k$ (Figure 1(e)).

- P6) Decrease-Constant pattern: existence of $\mathrm{s}_{\mathrm{n}-2} \mathrm{~s}_{\mathrm{n}-1} \mathrm{~S}_{\mathrm{n}}$ sequence, where:

○ $\mathrm{s}_{\mathrm{n}-2} \in \mathrm{R}_{\mathrm{i}}, \mathrm{s}_{\mathrm{n}-1} \in \mathrm{R}_{\mathrm{j}}, \mathrm{s}_{\mathrm{n}} \in \mathrm{R}_{\mathrm{k}}$ and $\mathrm{i}<\mathrm{j}, \mathrm{j}=\mathrm{k}$ (Figure 1(f)).

- P7) Constant-Increase pattern: existence of $s_{n-2} s_{n-1} s_{n}$ sequence, where:

○ $s_{n-2} \in R_{i}, s_{n-1} \in R_{j}, s_{n} \in R_{k}$ and $i=j, j>k$ (Figure $1(g)$ ).

- P8) Constant-Decrease pattern: existence of $s_{n-2} s_{n-1} s_{n}$ sequence, where:

○ $s_{n-2} \in R_{i}, s_{n-1} \in R_{j}, s_{n} \in R_{k}$ and $i=j, j<k$ (Figure 1(h)).

- P9) Constant pattern: existence of $\mathrm{s}_{\mathrm{n}-2} \mathrm{~s}_{\mathrm{n}-1} \mathrm{~s}_{\mathrm{n}}$ sequence, where:

○ $\quad s_{n-2} \in R_{i}, s_{n-1} \in R_{j}, s_{n} \in R_{k}$ and $i=j, j=k$ (Figure 1(i)).

where $s_{n}$ is $n$-th local extremum of $T_{n}$ windowed signal.

Features are defined by frequency of occurrence of each pattern in $T_{n}$. Therefore, feature vector $F=\left\{f_{1}, \ldots, f_{15}\right\}$ is constructed as below:

a) $f_{1}, f_{2}, f_{3}$ are number of local maxima happened in $R_{1}, R_{2}$, $\mathrm{R}_{3}$ range.

b) $f_{4}, f_{5}, f_{6}$ are number of local minima happened in $R_{1}, R_{2}$, $\mathrm{R}_{3}$ range.

c) $f_{7}$ to $f_{15}$ are number of happening P1 to P9 patterns.

This feature vector must be normalized by dividing to the number of local extrema (sum of $f_{1}$ to $f_{6}$ ). Feature vector $F$ then is used as the input of fuzzy logic system.

\subsection{Fuzzy Logic System}

A typical fuzzy logic system (Figure 2) contains two membership function sets for input and output and a fuzzy rule base. Inputs are evaluated by input membership functions and rules decide that the input how much belongs to outputs. 
(a)

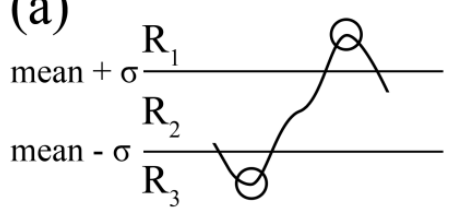

(d)

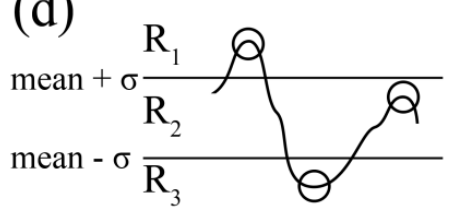

(g)

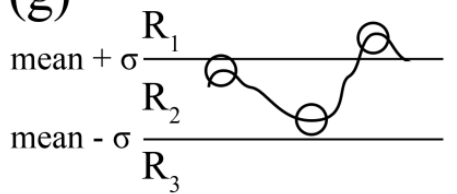

(b)

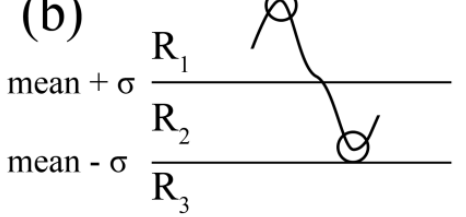

(e)

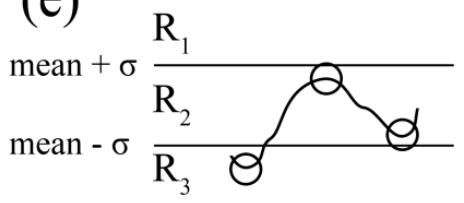

(h)

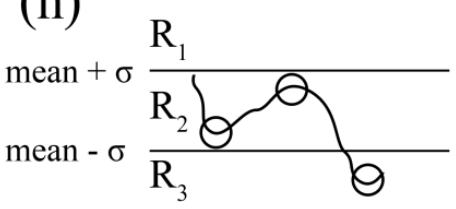

(c)

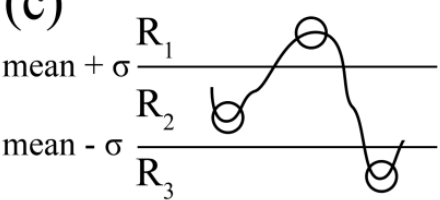

(f)

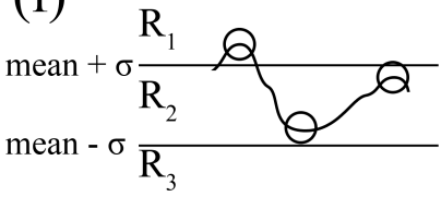

(i)

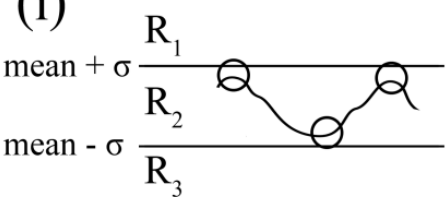

Figure 1. Example of nine patterns of SBLE method. a) Increase pattern b) Decrease pattern c) U-turn1 pattern d) Uturn2 pattern e) Increase-Constant pattern f) Decrease-Constant pattern g) Constant-Increase h) Constant-Decrease pattern i) Constant pattern.

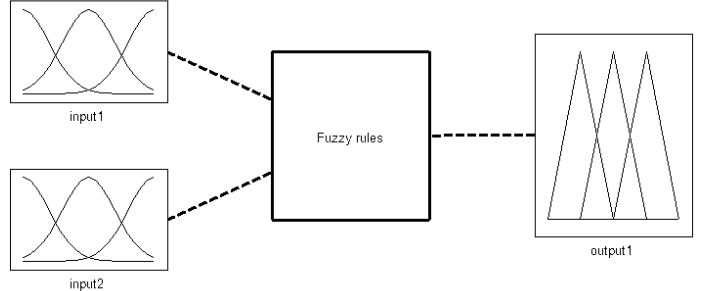

Figure 2. Simple schematic of fuzzy logic system.

Therefore, to design a fuzzy logic system, we first need membership functions of inputs and outputs.

Training dataset is employed to estimate input membership functions. For each patient histogram of values of each feature is extracted from ictal training dataset (histogram with 20 ranges). Then a Gaussian function is fitted on the histogram and after normalizing, these functions ( $\mathrm{mf} 1$ ) and compliment of them $(\mathrm{mf} 2)$ are used as input membership functions (Figure-3). Two compliment trapezoidal shaped functions are used as output membership functions (Figure-3).

Four rules are considered as rule bases in this study (a large number of rules will increase computational cost to find optimal rules, and as it will be shown, this number of rules is also effective). Format of these rules is shown in Figure 4.

For each rule antecedents, consequent and connection type between antecedents are unknown. Moreover, each rule has a weight that is used in defuzzification. Therefore, each rule has 18 unknown parameters and in fact there are 72 parameters that must be founded for four rules. To efficiently estimate these parameters Genetic algorithm (GA) is employed. A chromosome consist of 72 gens (60 binary gens for choosing rule antecedents, 4 binary gens for choosing connection type between antecedents, 4 binary gens for selecting consequents of rules and 4 float gens to find efficient weight of rules) are considered. 500 chromosomes per generation were considered where the number of generations is set as 50 . There are 20 chromosomes in each generation of the genetic mutation. The cost function $(\mathrm{CO})$ is defined as eq.2:

$\mathrm{CO}=\frac{\text { mean(FPR })+1}{\text { mean(sensitivity) }+1}$

Calculation of false positive rate (FPR) and sensitivity values will be described in evaluation section. The aim of GA is minimizing $\mathrm{CO}$ function by using the training dataset.

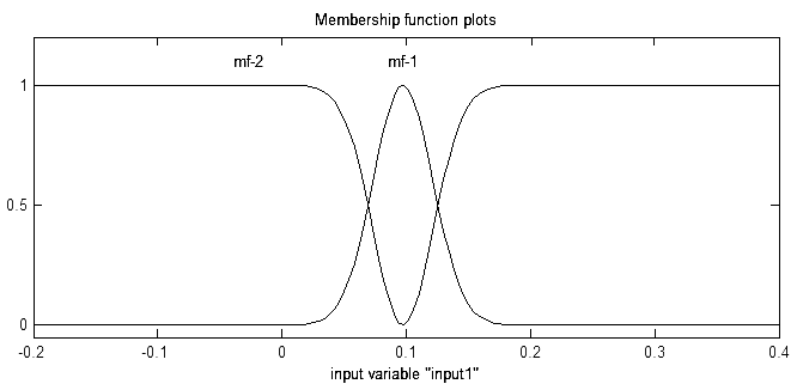

Membership function plots

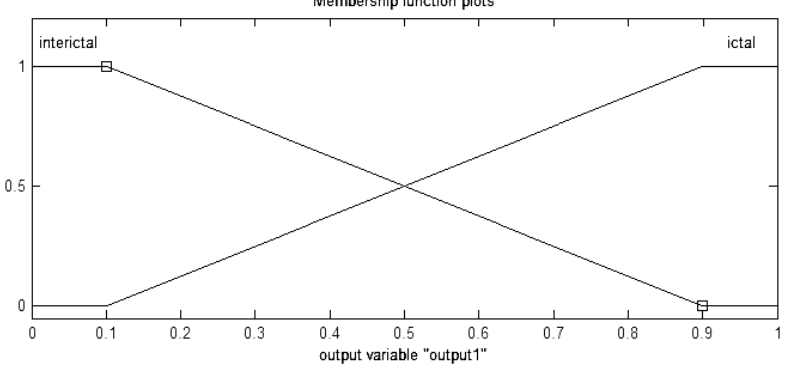

Figure 3. Examples of input (up) and output (down) membership functions.

GA extracts the rules that are needed for employment of fuzzy logic system. Before applying the decision-making method, output of fuzzy logic system needs to be prepared by a simple post-processing step. 


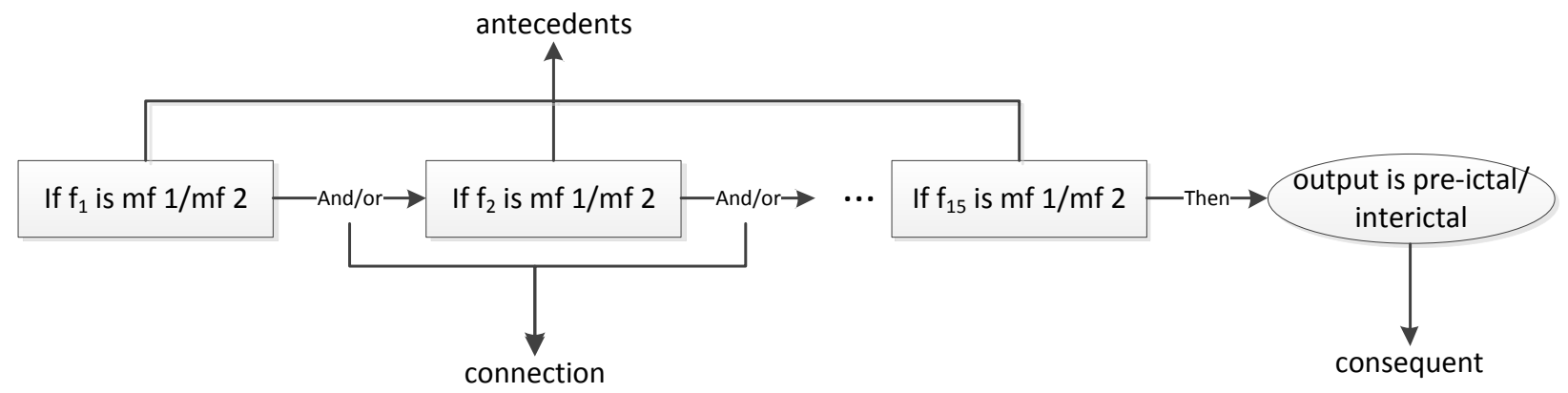

Figure 4. Template of rules containing antecedents, connection and consequent
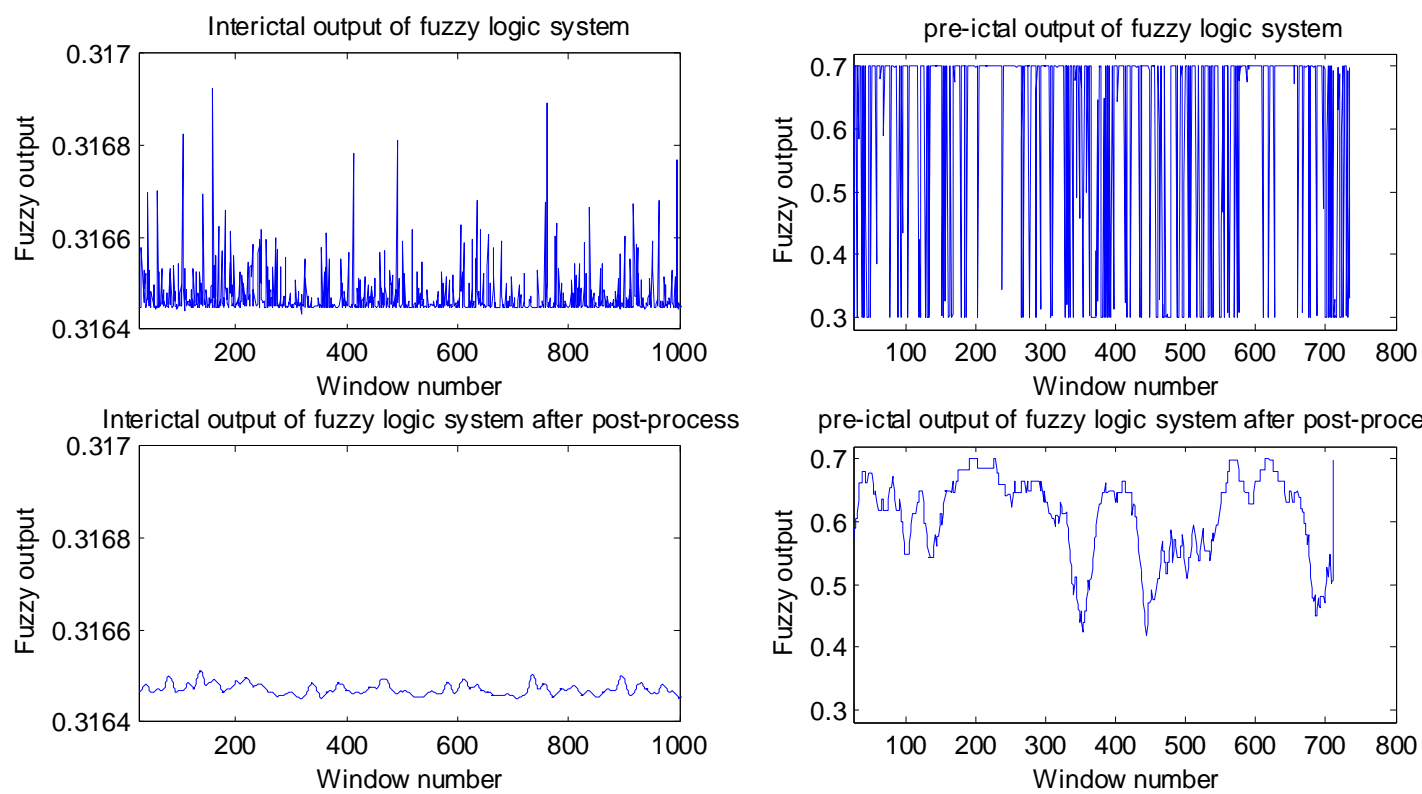

Figure 5. Output of fuzzy logic system for interictal and preictal inputs before and after post-processing. (Figure 5-1) Top-left plot is output of interictal inputs before smoothing. (Figure 5-2) Top-right plot is output of preictal inputs before smoothing. (Figure 5-3) Down-left plot is output of interictal inputs after smoothing. (Figure 5-4) Down-right plot is output of preictal inputs after smoothing.

\subsection{Post-Processing}

15 features that are extracted using SBLE method from $T_{n}$ windowed signal are used to feed the trained fuzzy system as the input. The output of the system indicates how much the input belongs to the preictal class. Because of short length of windows and lack of overlap between windows, output has large standard deviation and sharp peaks (Figure 5-1 and 5-2). Therefore, the output is smoothed by eq. 3 .

new output ${ }_{\mathrm{i}}=$ mean $\left(\right.$ output $_{\mathrm{i}-24}, \ldots$, output $\left.{ }_{\mathrm{i}}\right)$

Eq. 3 causes 120 seconds delay ( $24 * 5$-second windows). Figure 5-3 and 5-4 show the smoothed outputs. Epileptic seizures are predicted by applying a decision method based on a threshold on the smoothed outputs.

\subsection{Decision Making}

By using one-hour interictal data of training dataset output of fuzzy system $\mathrm{O}_{\mathrm{tr}}$ is obtained. FPR-Sensitivity evaluation requires changing the values of (200 thresholds that are generated by changing "i" from 1 to 200). Eq.4 is used to generate the thresholds:

$\operatorname{Tr}_{\mathrm{i}}=\max \left(\mathrm{O}_{\mathrm{t}}\right)-\frac{\max \left(\mathrm{O}_{\mathrm{t}}\right)-\min \left(\mathrm{O}_{\mathrm{t}}\right)}{100} * \mathrm{i} \quad 1 \leq \mathrm{i} \leq 200$

Features of all $T_{n}$ windowed signals are extracted and by using a fuzzy system the output is calculated. There are two rules and one flag for decision making:

1) If the output is less than the threshold and flag is zero, then a seizure will not happen for 30 minutes and the flag is zero for 30 minutes.

2) If the output is larger than the threshold for 30 minutes, then a seizure will occur within 30 minutes and the flag is one for 30 minutes.

For example in Figure 6-1 the output in the first 30 minutes interval is higher the threshold so in the next 30 minutes interval a seizure will happen. In Figure 6-2 U-shaped points are under the threshold so the next 30 minutes interval will be seizure free. 
1)

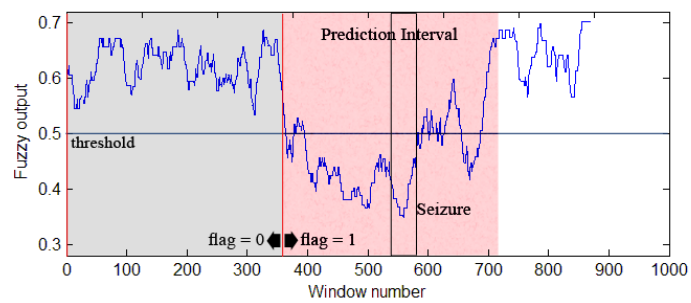

2)

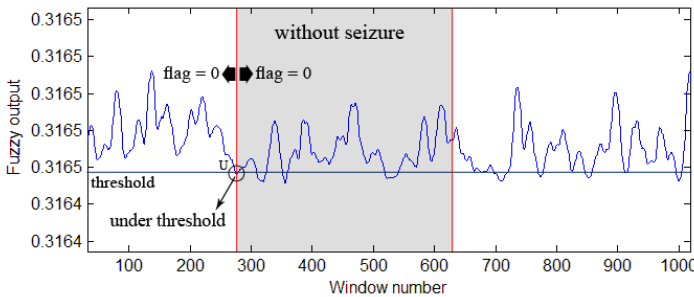

Figure 6. Sample of decision making. In top Figure, in the first 30 minutes there is not any point below the threshold so in the next 30 minutes a seizure will occur. Point $U$ in down Figure is the first point below the threshold so in the next 30 minutes no seizure will happen. In Figure 6-1 there are points lower than the threshold in prediction interval but since "flag $=1$ " these points will be ignored. Figure 6-2 shows an interval without any seizure that is caused by point $U$, although there are many other points lower than the threshold after $U$, that cause other seizurefree intervals after $U$.

For each threshold two parameters, FPR and sensitivity are calculated as eq.5 and eq.6:

$\mathrm{FPR}=\frac{\text { number of false alarms }}{\text { time in hours }}$

Sensitivity $=\frac{\text { number of true alarms }}{\text { number of seizures }}$

A false alarm is occurred when there is no point under the threshold in 30 minutes interval of interictal output. True alarm is existence of a 30 minutes interval of output before a seizure that does not have any point under threshold.

In section 3-3, a cost function in genetic algorithm used the average values of FPR and sensitivity in different thresholds that are extracted from interictal and preictal training dataset. Figure 8 shows the block diagram of the proposed method for epileptic seizure prediction

\section{RESULTS}

The proposed method is evaluated by two approaches. First approach is patient-dependent, in which, all the training dataset is used to extract rules and membership functions. In this approach, membership functions for each patient are extracted from preictal dataset of the same patient and GA utilizes the whole training dataset. In the second approach, leave one out (LOO), leave one patient out technique is used. The generalizability of the method must be evaluated by testing the method without using the patient data for training of the system. In LOO technique, first, the patient data is excluded and is only used for testing. Then, the remaining data are used for training of the system. Membership functions for the test patient are randomly selected from the other patient membership functions. LOO technique evaluates the generalizability of the method.
Table 1 shows the result of the proposed method in patientdependent approach for test dataset of all the 21 patients of Freiburg database in two different thresholds. In first threshold, FPR is zero that means no false alarm in the all interictal interval and a high specificity. In this threshold a sensitivity of $84.84 \%$ for all patients is achieved. By decreasing the threshold value, the numbers of false alarm increases (that means higher FPR) but the sensitivity will increases. In the second threshold, a FPR of 0.1 and sensitivity of $93.93 \%$ is achieved.

Table 1. Results of all the 21 patients, using patientdependent approach for testing

\begin{tabular}{|c|c|c|c|c|c|}
\hline \multirow[b]{2}{*}{ 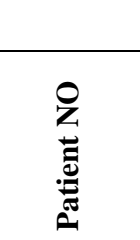 } & \multirow[b]{2}{*}{ 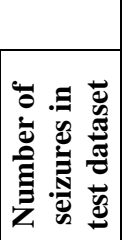 } & \multicolumn{2}{|c|}{$\operatorname{Tr}_{190}$} & \multicolumn{2}{|c|}{$T r_{191}$} \\
\hline & & 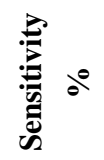 & $\frac{a}{|c|}$ & $\sum_{\substack{0 \\
0}}$ & 点 \\
\hline Patient 1 & 3 & 66.67 & 0 & 100 & 0.4166 \\
\hline Patient 2 & 2 & 50 & 0 & 100 & 1 \\
\hline Patient 3 & 4 & 100 & 0 & 100 & 0 \\
\hline Patient 4 & 4 & 100 & 0 & 100 & 0 \\
\hline Patient 5 & 4 & 75 & 0 & 100 & 0.0520 \\
\hline Patient 6 & 2 & 100 & 0 & 100 & 0 \\
\hline Patient 7 & 2 & 50 & 0 & 100 & 0.2604 \\
\hline Patient 8 & 1 & 100 & 0 & 100 & 0 \\
\hline Patient 9 & 4 & 100 & 0 & 100 & 0 \\
\hline Patient 10 & 4 & 75 & 0 & 75 & 0 \\
\hline Patient 11 & 3 & 66.67 & 0 & 66.67 & 0.1041 \\
\hline Patient 12 & 3 & 100 & 0 & 100 & 0 \\
\hline Patient 13 & 1 & 0 & 0 & 100 & 0.4166 \\
\hline Patient 14 & 3 & 100 & 0 & 100 & 0 \\
\hline Patient 15 & 3 & 100 & 0 & 100 & 0 \\
\hline Patient 16 & 4 & 100 & 0 & 100 & 0 \\
\hline Patient 17 & 4 & 75 & 0 & 75 & 0.1562 \\
\hline Patient 18 & 4 & 50 & 0 & 75 & 0.2083 \\
\hline Patient 19 & 3 & 100 & 0 & 100 & 0 \\
\hline Patient 20 & 4 & 100 & 0 & 100 & 0 \\
\hline Patient 21 & 4 & 100 & 0 & 100 & 0 \\
\hline \multicolumn{2}{|c|}{ Mean } & 84.848 & 0 & 93.939 & 0.1009 \\
\hline
\end{tabular}

Table 2 reports the result of testing the method by using LOO technique. As is seen, the sensitivity decreased and in FPR of 0.048 , a sensitivity of $80.45 \%$ is achieved. Reducing sensitivity is related to not using proper membership functions for test patient and not using whole training dataset. Nevertheless, as we have seen, the sensitivity for a large number of patients in FPR of 0 is $100 \%$. However, patients who do not respond well to the method of patient-dependent testing also do not have an appropriate response to LOO testing technique. EEG Freiburg dataset has some limitations 
including lack of information about the patients' medication and a few hours of preictal data [16]. Therefore, we cannot comment on the reason for the poor or good response.

Table 2. Result of all 21 patients, using LOO technique approach for testing.

\begin{tabular}{|c|c|c|c|}
\hline \multirow[b]{2}{*}{ 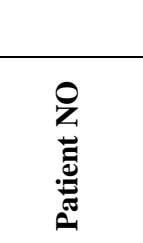 } & \multirow[b]{2}{*}{ 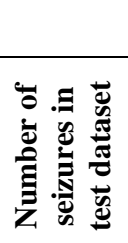 } & \multicolumn{2}{|c|}{$T r_{190}$} \\
\hline & & 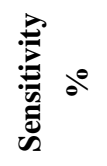 & 总 \\
\hline Patient 1 & 4 & 75 & 0 \\
\hline Patient 2 & 3 & 66.67 & 0 \\
\hline Patient 3 & 5 & 60 & 0 \\
\hline Patient 4 & 5 & 100 & 0 \\
\hline Patient 5 & 5 & 80 & 0 \\
\hline Patient 6 & 3 & 66.67 & 0 \\
\hline Patient 7 & 3 & 33.33 & 0 \\
\hline Patient 8 & 2 & 100 & 0 \\
\hline Patient 9 & 5 & 100 & 0 \\
\hline Patient 10 & 5 & 80 & 0 \\
\hline Patient 11 & 4 & 75 & 1 \\
\hline Patient 12 & 4 & 75 & 0 \\
\hline Patient 13 & 2 & 0 & 0 \\
\hline Patient 14 & 4 & 100 & 0 \\
\hline Patient 15 & 4 & 100 & 0 \\
\hline Patient 16 & 5 & 100 & 0 \\
\hline Patient 17 & 5 & 60 & 0 \\
\hline Patient 18 & 5 & 60 & 0 \\
\hline Patient 19 & 4 & 100 & 0 \\
\hline Patient 20 & 5 & 100 & 0 \\
\hline Patient 21 & 5 & 100 & 0 \\
\hline & & 80.459 & 0.0488 \\
\hline
\end{tabular}

\section{CONCLUSIONS}

The advantage of the proposed method over other classification techniques, such as ANN, is that it provides the output as a linear regression time series rather than integer values representing classes. This aspect of fuzzy logic system is valuable, as it allows the performance analysis within the framework of seizure prediction characteristics [21]. Moreover, fuzzy logic system is capable to accommodate human knowledge and reasoning as well as machine learning capabilities [22]. By using the proposed method we achieved results that demonstrate the applicability of fuzzy logic system to combine features for seizure prediction. First evaluation method and its results show the ability of the features and system to classify preictal and interictal states. Then, LOO technique evaluates the generalizability of the method. It achieved a sensitivity of $100 \%$ with false alarm rate of zero on 10 patients of Freiburg database. Therefore this method can work perfectly on some cases and situations. Overall, the results show that the system has a high specificity (low FPR) in a conventional sensitivity.

The proposed method uses data to for training, so it can be performed automatically and does not require initialization by user. In LOO evaluates there is not stability to membership functions that is selected randomly. Thus, finding others approaches to estimate membership functions that do not require preictal data may help robustness of the method.

Using fuzzy logic system to combine SBLE features has led to needing train dataset. Finding others combination methods that do not need to train dataset can be considered as the future scope of the idea.

\section{REFERENCES}

[1] S. Shorvon, Handbook of epilepsy treatment, Blackwell Pub, 2006.

[2] E. Reynolds, R. Elwes and S. Shorvon, "Why does epilepsy become intractable?: prevention of chronic epilepsy," The Lancet, vol. 322, pp. 925-954, 1983.

[3] F. Mormann, R. Andrzejak, C. Elger and K. Lehnertz, "Seizure prediction: the long and winding road," Brain, vol. 130, pp. 314-333, 2007.

[4] C. Elger and D. Schmidt, "Modern management of epilepsy: A practical approach," Epilepsy \& Behavior, vol. 12, pp. 501-539, 2008.

[5] D. Kugiumtzis and P. Larsson, "Linear and nonlinear analysis of EEG for the prediction of epileptic seizures," in Proceeding of the 1999 Workshop "Chaos in Brain?", Singapore, 2000.

[6] S. Viglione and G. Wlsh, "Proceedings: Epileptic seizure prediction," Electroencephalogr Clin Neurophysiol, vol. 39, pp. 435-436, 1975 .

[7] Z. Rogowski, I. Gath and E. Bental, "On the prediction of epileptic seizures," Biol Cybern, vol. 42, pp. 9-15, 1981.

[8] Y. Salant, I. Gath and O. Henriksen, "Prediction of epileptic seizures from two-channel EEG," Med Biol Eng Comput, vol. 36, pp. 549-556, 1998.

[9] L. Iasemidis, J. Sackellares, H. Zaveri and W. Williams, "Phase space topography and the Lyapunov exponent of electrocorticograms in partial seizures," Brain Topogr, vol. 2, pp. 187-201, 1990.

[10] R. Costa, P. Oliveira, G. Rodrigues, B. Leitao and A. Dourado, "Epileptic seizure classification using neural networks with 14 features," pp. 281-288, 2008.

[11] "Freiburg seizure prediction database," 2007. [Online]. Available: http://epilepsy.uni-freiburg.de/freiburgseizure-prediction-project/eeg-database.

[12] N. Moghim and D. W. Corne, "Predicting Epileptic Seizures in Advance," PLoS ONE, vol. 9(6), p. e99334, 2014.

[13] P. Ghaderyan, A. Abbasi and M. Sedaaghi, "An efficient seizure prediction method using KNN-based undersampling and linear frequency measures," Journal of Neuroscience Methods, 2014.

[14] F. Mormann, T. Kreuz, C. Rieke, R. Andrzejak, A Kraskov, P. David, C. Elger and K. Lehnertz, "On the 
predictability of epileptic seizures," Clin Neurophysiol, vol. 116, pp. 569-87, 2005.

[15] Geva and D. Kerem, "Forecasting generalized epileptic seizures from the EEG signal by wavelet analysis and dynamic unsupervised fuzzy clustering," IEEE Trans. Biomedical Engineering, vol. 45, pp. 1205-1216, 1998.

[16] P. Mirowski, D. Madhavan, Y. LeCun and R. Kuzniecky, "Classification of patterns of EEG synchronization for seizure prediction," Clin Neurophysiol, vol. 120, pp. 1927-40, 2009.

[17] Y. Park, L. Luo, K. Parhi and T. Netoff, "Seizure prediction with spectral power of EEG using costsensitive support vector machines," Epilepsia, vol. 52, pp. 1761-70, 2011.

[18] Aarabi and B. He, "Seizure prediction in intracranial EEG: A patient-specific rule-based approach," in Med. Biol. Soc., Boston, 2011.
[19] Aarabi, R. Fazel-Rezai and Y. Aghakhani, "A fuzzy rulebased system for epileptic seizure detection in intracranial EEG," Clinical Neurophysiology, vol. 120, pp. 1648-1657, 2009.

[20] Rabbi, L. Azinfar and R. Fazel-Rezai, "Seizure Prediction Using Adaptive Neuro-Fuzzy Inference System," in IEEE EMBS, Osaka, 2013.

[21] T. Maiwald, M. Winterhalder, R. AschenbrennerScheibe, H. Voss, A. Schulze-Bonhage and J. Timmer, "Comparison of three nonlinear seizure prediction methods by means of the seizure prediction characteristic," Physica D, vol. 194, pp. 357-368, 2004.

[22] L. Tsoukalas and R. Uhrig, Fuzzy and Neural Approaches in Engineering, 1996. 